Wenig hoffnungsvoll scheint die Verwendung als Ersatz für die bekannten Kühlmaschinen nach dem bisher Erreichten zu sein. Diese haben im Gebiet der kleinen Druckverhältnisse bessere Nutzeffekte. Trotzdem sind Fälle denkbar, wo ein Wirbelrohr wegen des einfachen Aufbaues in Frage kommt (z. B. Kühlung vor Ort im Bergwerk).
Anders liegen die Verhältnisse bei tiefen Ausgangstemperaturen und bei hohen Druckverhältnissen. Im Gebiet der Gasverflüssigung wird sich das Wirbelrohr als wirksamer Kälteapparat dem Drosseleffekt überlegen zeigen. Für diese Anwendung auf Tiefsttemperaturen sind weitere Messungen geplant, über die später berichtet werden soll.

\title{
Über natürliche Zerfallsspektren von Benzolderivaten und ihren Zusammenhang mit dem Phosphorescenzleuchten
}

\author{
Von H. Schüler und A. Woeldike \\ Aus dem Kaiser-Wilhelm-Institut für Physik, Hechingen \\ (Naturforschg. 1, 214-217 [1946]; eingegangen am 5. April 1946)
}

\begin{abstract}
Bei Beobachtung von Emissionsspektren organischer Substanzen zeigt sich an Mono- und Diderivaten des Benzols, daß angeregte Molekülzustände existieren, aus denen heraus die Moleküle von selbst zerfallen und zwar so, daß eines der Bruchstücke im angeregten Zustand zurückbleibt. Bei den untersuchten Substanzen wird beim Zerfall der Phenylrest angeregt, und zwar sein Bindungselektron zum abgerissenen Substituenten hin. Dadurch entstehen zwei Typen von Spektren (blau und grün), deren Zusammenhänge diskutiert werden. Auffallende Ähnlichkeiten zwischen diesem Zerfallsleuchten in der Gasphase und den Phosphorescenzerscheinungen in der festen Phase lassen den Schluß zu, daß das Phosphorescenzleuchten in den betrachteten Fällen von Störstellen im Krystall herrührt, an denen zwischen Substituent und Phenylrest eine derartig starke Lockerung der Bindung eingetreten ist, daß man von einer Anregung eines Molekülbruchstückes sprechen muß. Diese eindeutige Zuordnung eines Leuchtprozesses in der festen Phase zu einem Leuchtprozeß in der gasförmigen Phase gestattet einen Einblick in die Vorgänge der festen Phase.
\end{abstract}

$\mathrm{D}$ ie natürlichen Zerfallsspektren organischer Moleküle, deren Existenz bereits in früheren Untersuchungen $^{1}$ von den Verfassern festgestellt wurde, beweisen, daß es bei den vielatomigen Molekülen Anregungszustände gibt, in denen die aufgenommene Elektronenenergie dazu benutzt wird, das Molekül zu zerlegen und mindestens eines von den Bruchstücken in einem angeregten Zustand zurückzulassen. Das Spektrum dieses angeregten Molekülbruchstücks ist dann der unmittelbare Ausdruck für den eben dargelegten Zerfall. Voraussetzung dabei ist natürlich, daß durch die experimentellen Manipulationen alle gewaltsamen Zerstörungen der Moleküle vermieden sind und daß tatsächlich nur der elementare Zerfallsprozeß auftritt ${ }^{2}$. Während bei den zweiatomigen Molekülen der Ablauf solcher Zerfallsprozesse von vornherein eindeutig festgelegt und schon verschiedentlich

1 H. Schüler u. A. Woeldike. Physik. Z. 43, 17 [1942]. beobachtet ist, kann man bei den vielatomigen Molekülen die Bruchstücke, in die das Molekül zerfallen wird, und die Bruchstücke, die -angeregt zurückbleiben, a priori nicht festlegen, ganz abgesehen davon, daß bei den organischen Molekülen solche Zerfallsprozesse bisher noch nicht diskutiert worden sind. Es ist nun an einer Reihe von Benzolderivaten der natürliche Zerfall studiert worden. In Tab. 1 sind die Resultate wiedergegeben. Die ersten Substanzen von Toluol bis Benzoesäure zeigen neben ihren spezifischen Emissionsspektren im Ultraviolett, die auf die Anregung der 6r-Elektronen des Benzolringes zurückzuführen sind, alle im Sichtbaren das gleiche Spektrum. Wegen seiner Farbe haben wir es als das ,blaue Spektrum“ bezeichnet. Die Wellenlängen seiner markantesten Banden sind, wie früher bereits mitgeteilt: $\lambda=3713$; 3970,$3 ; 4263,0 ; 4597,4 ; 4985,4$; 5420,3 AE. Ein Bild

2 H. Schül er u. A. Woeldike, Physik. Z. 42, 390 [1941]. 


\begin{tabular}{|c|c|c|}
\hline \multirow[b]{2}{*}{ Substanz } & \multicolumn{2}{|c|}{ geschätzte Intensität des } \\
\hline & $\begin{array}{c}\text { blauen } \\
\text { Spektrums }\end{array}$ & $\begin{array}{c}\text { grünen } \\
\text { Spektrums }\end{array}$ \\
\hline 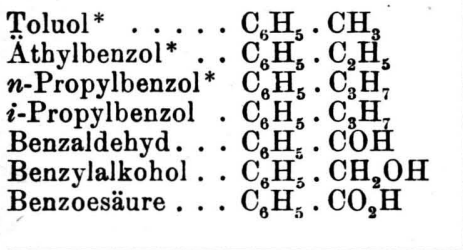 & $\overline{-}$ & $\begin{array}{l}\overline{ } \\
\ldots \ldots \\
\ldots \ldots \\
\ldots \ldots \\
\ldots \ldots\end{array}$ \\
\hline $\begin{array}{l}\text { Chlorbenzol ... } \mathrm{C}_{6} \mathrm{H}_{5} \cdot \mathrm{Cl} \\
\text { Brombenzol ... } \mathrm{C}_{6} \mathrm{H}_{5} \cdot \mathrm{Br} \\
\text { Phenol . . . } \mathrm{C}_{6} \mathrm{H}_{5} \cdot \mathrm{OH} \\
\text { Benzonitril ... } \mathrm{C}_{6} \mathrm{H}_{5} \cdot \mathrm{CN} \\
\text { Anilin . . . . } \mathrm{C}_{6} \mathrm{H}_{5} \cdot \mathrm{NH}_{2} \\
\text { Anisol . . . } \mathrm{C}_{6} \mathrm{H}_{5} \cdot \mathrm{OCH}_{3}\end{array}$ & $\begin{array}{l}\ldots \\
\cdots \\
\cdots\end{array}$ & $\begin{array}{l}. \cdots \\
. \cdots \\
. \cdots \\
. \cdots \\
. \cdots \\
. \cdots \\
\cdots\end{array}$ \\
\hline $\begin{array}{l}\text { Nitrobenzol }{ }^{* *} \ldots \mathrm{C}_{6} \mathrm{H}_{5} \cdot \mathrm{NO}_{2} \\
\text { Nitrotoluol }^{* *} \ldots \mathrm{CH}_{3} \cdot \mathrm{C}_{6} \mathrm{H}_{4} \cdot \mathrm{NO}_{2}\end{array}$ & $\cdots$ & $\cdots$ \\
\hline
\end{tabular}

Tab. 1. Emissionsspektren von Benzol-Monoderivaten.

* Das Intensitätsverhältnis des grünen zum blauen Spektrum ist so dargestellt, wie es sich bei einem mittleren Substanzdruck ergibt. Beide Spektren sind wiedergegeben in Physik. Z. 43, 17 [1942], Tafel V, Spektrum a, bzw. Tafel VI, Spektrum b-d, und Tafel VII.

** Zerfallsspektrum vom Nitrobenzol und Nitrotoluol. Physik. Z. 45, 171 [1944], identisch mit dem $\mathrm{NO}_{2}$-Spektrum.

dieses Spektrums wurde in einer früheren Arbeit ${ }^{1}$ (Tafel VII) wiedergegeben. Durch die Länge der horizontalen Striche in Tab. 1 soll ungefähr die Intensität des blauen Spektrums angedeutet werden, wie sie sich bei möglichst gleichen Versuchsbedingungen ergibt. Daß es sich bei diesem blauen Spektrum nicht um eine allen hier untersuchten Substanzen gemeinsame Verunreinigung handelt, ergibt sich aus charakteristisch abweichenden Befunden an den Diderivaten des Benzols, worüber weiter unten noch zu berichten sein wird. Danach ist man also berechtigt, dieses blaue Spektrum als ein Zerfallsspektrum anzusehen, das von der Anregung eines allen erwähnten Monoderivaten gemeinsamen Bruchstückes herrührt. Dieses Bruchstück kann nur der Phenylrest $\left(\mathrm{C}_{6} \mathrm{H}_{5}\right)$ sein. Das Nichterscheinen dieses blauen Spektrums in der Reihe von Chlorbenzol bis Anisol muß dann zunächst einmal so erklärt werden, daß beim etwaigen Zerfall nicht mehr genügend Energie für die Anregung des Phenylrestes zur Verfügung steht. Etwas komplizierter werden die Zerfallserscheinungen noch dadurch, daß bei einigen Benzolderivaten, nämlich bei Toluol, Äthylbenzol und $n$-Pro- pylbenzol, ein zweites Zerfallsspektrum auftritt, das wir wegen seines Aussehens als ,grünes Spektrum" bezeichnet haben. Es ist in ${ }^{1}$, Tafel I, Spektrum a, wiedergegeben. Die Wellenlängen der Hauptkanten sind: $\lambda=4477,5 ; 4492,7 ; 4654,6$; 4674,$8 ; 4888 ; 4902 \mathrm{AE}$. Zwischen dem grünen und dem blauen Zerfallsspektrum bestehen nun insofern Zusammenhänge, als es möglich ist, durch Variation des Druckes im Entladungsrohr das eine Spektrum auf Kosten des anderen intensiver zu machen. Der Druckbereich, in dem die Röhre arbeitet, liegt zwischen 0,1 und $2 \mathrm{~mm} \mathrm{Hg}$. So beobachtet man z. B. am Athylbenzol bei geringem Druck das grüne Spektrum, bei höherem Druck das blaue Spektrum. Auch für das grüne Zerfallsspektrum muß der Phenylrest maßgeblich verantwortlich sein.

Zusammenfassend läßt sich also sagen, daß bei den Monoderivaten zwei Zerfallsspektren auftreten, die der Anwesenheit des Phenylrestes zuzuschreiben sind. Welche spezifische Konfiguration für jedes der beiden Spektren vorliegt, läßt sich zur Zeit noch nicht entscheiden. Die Tatsache jedoch, daß bereits bei einer geringen Druckänderung das eine Zerfallsspektrum in das andere übergeht, läßt vermuten, daß beide Spektren aus dem gleichen Anregungszustand des Moleküls hervorgehen und vom gleichen Molekülbruchstück stammen. Die Unterschiedlichkeit der Spektren wäre dann bedingt durch die Verschiedenheit der Schwingungstypen des Bruchstückes, die sich beim Zerfall des Moleküls herausbilden können. Für die Deutung ist es wesentlich, darauf hinzuweisen, daß für das blaue Spektrum sich eine $\Delta y$-Folge von 1745,1729 , 1706, 1692 und $1609 \mathrm{~cm}^{-1}$ ergibt, deren Verlauf auf Schwingungen im Grundzustand hinweist (die Differenzen nehmen nach längeren Wellen zu ab). Das grüne Spektrum weist eine Hauptschwingung von $\Delta \nu \sim 940 \mathrm{~cm}^{-1}$ auf. Dieses Spektrum sieht dem ultravioletten Benzolspektrum in bezug auf die äußere Erscheinung seiner Banden ähnlich. Die Tatsache, daß die hier beobachteten Spektren des „Phenylrestes" im Sichtbaren liegen, während die energieärmsten Elektronenübergänge vom Benzol und seinen Derivaten im Ultraviolett zu beobachten sind, deutet darauf hin, daß beim natürlichen Zerfall der Anregungszustand anderer Elektronen nachgewiesen wird als bei der Anregung der Gesamtmoleküle. Die Annahme liegt nahe, daß bei der Trennung des Substituenten vom Benzolring eines der beiden Bindungselektronen in einem an- 
geregten Zustand zurückbleibt, und daß bei den bisher diskutierten Substanzen die Energie auf das Elektron übertragen wird, das dem Benzolring angehört. Beim Nitrobenzol und Nitrotoluol, die in Tab. 1 an letzter Stelle stehen, muß dagegen eine Anregung des Bindungselektrons des Substituenten $\left(\mathrm{NO}_{2}\right)$ angenommen werden, denn hier tritt beim natürlichen Zerfall ein kontinuierliches Spektrum des $\mathrm{NO}_{2}$ auf, und weder das grüne, noch das blaue Zerfallsspektrum werden beobachtet ${ }^{3}$.

Die Befunde an Diderivaten des Benzols lassen sich im gleichen Sinn wie die Beobachtungen an den Monoderivaten deuten. Es liegen Untersuchungen über $p$-, $o$ - und $m$-Xylol ${ }^{1}$ und Toluylaldehyd vor. Auch hier werden blaue und grüne Spektren gefunden (s. Tab.2). Da bei diesen Substanzen noch ein zweiter Substituent vorhanden ist, der seine Lage zur Abreißstelle ändert ( $p$-, o-, $m$-Stellung), so ergibt sich im Gegensatz zu den Monoderivaten eine Vielheit von grünen bzw. blauen Spektren, die durch Kursivsatz der Worte grün bzw. blau angedeutet werden soll. In ${ }^{1}$, Tafel V (Spektren b bis d) sind die grünen Spektren der Xylole wiedergegeben. Man sieht, daß die Stellungsänderung des verbleibenden $\mathrm{CH}_{3}$-Substituenten zur Abreißstelle das Aussehen der Spektren selbst wohl ändert, daß aber die Lage der Spektren, die ja durch den gleichen Elektronensprung bestimmt wird, energetisch praktisch die gleiche ist. Analog zu den Monoderivaten werden auch hier die grünen Spektren bei geringerem Druck und die blauen Spektren bei etwas höherem Druck beobachtet. Der Vergleich des blauen $p$-Xylol-Spektrums ${ }^{1}$ mit dem blauen Spektrum der Monoderivate zeigt, daß die gleichen Bandengruppen, aber mit einer geringen Verschiebung nach Violett zu, auftreten, was auch ein Zeichen dafür ist, daß es sich bei dem Zerfallsspektrum nicht um eine gemeinsame Verunreinigung handeln kann. Für das blaue Zerfallsspektrum des p-Toluylaldehyds gibt es von vornherein 2 Möglichkeiten; entweder wird der $\mathrm{CH}_{3}$-Substituent oder die $\mathrm{COH}$ Gruppe abgetrennt. In dem Fall der $\mathrm{COH}-A b t r e n-$ nung muß ein blaues Spektrum erscheinen, das mit dem des $p$-Xylols identisch ist. Der Befund beweist, daß das tatsächlich der Fall ist.

Es muß noch erwähnt werden, daß die hier beschriebenen blauen und grünen Spektren bereits von McVicker und Mitarbeitern ${ }^{4}$ mit Hilfe der Tesla-Anregung erzeugt worden sind. Diese Ar-

\footnotetext{
${ }^{3}$ H.Schüler u. A. Woeldike, Physik. Z. 45, 171 [1944].
}

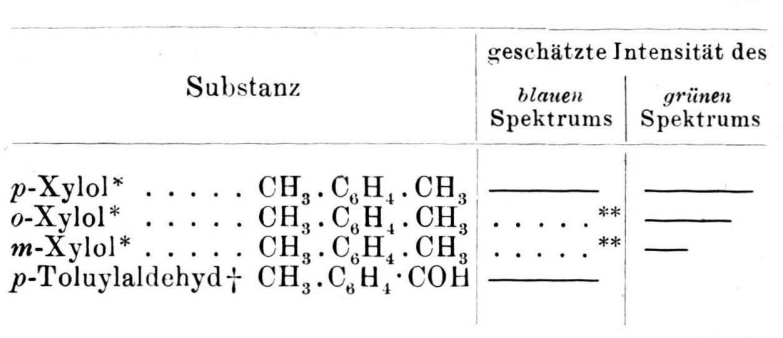

Tab. 2. Emissionsspektren von Benzol-Diderivaten.

* Grüne Spektren wiedergegeben in Physik. Z. 43, 17 [1942], Tafel V, Spektren b-d.

** Diese Spektren sind nur subjektiv beohachtet und nicht photographiert worden, da zu wenig Substanz zur Verfügung stand.

$\dagger$ Blaues Spektrum identisch mit dem des $p$-Xylol. Physik. Z. 43, 17 [1942], Tafel VI.

beiten, die zum Teil mit komplizierteren Substanzen ausgeführt wurden, sind als eine erste Orientierung aufzufassen. Es wird von ihnen weder zwischen grünen und blauen Spektren unterschieden, noch ist die Deutung dieser Spektren als Zerfallsspektren gegeben worden.

Die Beobachtung der oben beschriebenen, im Sichtbaren liegenden Zerfallsspektren bei Monound Diderivaten des Benzols lenkt nun die Aufmerksamkeit auf eine andere Gruppe von Leuchterscheinungen bei den gleichen Substanzen: die Phosphorescenzspektren im festen Zustand. Die hierbei beobachteten Spektren liegen nicht nur im gleichen Spektralbereich wie die Zerfallsspektren, sondern es zeigen sich auch derartig große Ähnlichkeiten in bezug auf Lage der Banden und Schwingungsdifferenzen, daß für beide Fälle die gleichen Mechanismen im Molekül angenommen werden müssen. A. Kronenberger ${ }^{5}$ hat bereits bei seinen Phosphorescenzuntersuchungen an Xylolen auf die Gleichartigkeit der Spektren im Hinblick auf M c Vickersche Arbeiten aufmerksam gemacht. So gibt er u. a. beim $p$-Xylol folgende Bandenkanten an: $\lambda$ 3940/3964, 4224/4256, 4550/ $4582 \mathrm{AE}$. Wir finden für die gasförmige Phase die entsprechenden Werte: $\lambda=3945,4235,4560 \mathrm{AE}$. Es sind noch zahlreiche weitere Übereinstimmungen auf Grund der Angaben von E. Goldstein ${ }^{6}$, der sich ebenfalls mit der Untersuchung von Phosphorescenzspektren bei Benzolderivaten beschäftigt hat, festzustellen. So wird z. B. immer auf die

4 W. H. M c Vicker, J. K. M a r sh u. A. W. Ste w a t, J. chem. Soc. [London] 123, 2147 [1923]; 125, 1743 [1924].

5 Z. Physik 63, 494 [1930].

6 Physik. Z. 12, 614 [1911]. 
große Intensität der ,blauen Serien“ des Benzaldehyds hingewiesen, während wir in der Glimmentladung ebenfalls beim Benzaldehyd das stärkste Zerfallsspektrum festgestellt haben (s. Tab.1).

Es liegt hier also folgende Situation vor: Bei einer Anzahl organischer Substanzen sind die Leuchterscheinungen in der Glimmentladung mit den Phosphorescenzerscheinungen der festen Phase als identisch anzusehen, wobei die gasförmige Phase die Aussage gestattet, daß das beobachtete Leuchten von einem natürlichen Zerfallsprozeß des angeregten Moleküls herrührt. Für die Phosphorescenzerscheinung ergibt sich dann in der festen Phase folgendes Bild: Im Krystall sind Störstellen vorhanden, in denen eine Lockerung der Bindung zwischen Substituent und Phenylring eingetreten ist. Diese Lockerung muß groß sein, da im Phosphorescenzleuchten praktisch nichts mehr von einem Einfluß des Substituenten zu bemerken ist.

Damit ist eindeutig eine Aussage über den Molekülzustand gemacht, auf den das Phosphorescenzleuchten zurückzuführen ist. Im allgemeinen besteht ja dieses Leuchten aus breiten kontinuierlichen Bändern, die eine Zuordnung zu diskreten Energiezuständen von Molekülen bzw. Atomen immer mehr oder weniger hypothetisch machen. Die Fälle liegen bei den hier besprochenen Substanzen insofern günstig, als die Phosphorescenz- banden noch ziemlich gut definiert sind und eine einwandfreie Zuordnung zu Leuchtprozessen in der Gasphase möglich ist. Bemerkenswert ist nun die Feststellung, daß am Leuchtprozeß der Phosphorescenz in unseren Fällen gar nicht die Gesamtmoleküle beteiligt sind, sondern nur Bruchstücke derselben. Während die Beobachtung der Zerfallsspektren - also in der Gasphase - eine Energiezufuhr an das Gesamtmolekül verlangen, die sich aus der Abtrennungsenergie des Substituenten und der Anregungsenergie des Phenylrestes zusammensetzt und sicher unterhalb von $\lambda=2500 \mathrm{AE}$. liegt, wird in der Phosphorescenz nur eine Energie gefordert, die praktisch gleich der Anregung des Phenylrestes ist. So ist es verständlich, daß Gold s te in bereits mit Licht, das durch Uviolglas gefiltert ist (kürzeste Wellenlänge $\lambda=2600 \mathrm{AE}$.), die hier beschriebene Phosphorescenz beobachten kann. Die Frage, ob die hier gegebene Erklärung für das Phosphorescenzleuchten sich nur auf organische Moleküle bezieht, oder ob auch bei den Substanzen anderer Phosphorenklassen (z. B. Zinksulfidkrystalle ${ }^{\tau}$ ) analoge Deutungen zutreffen, soll im Rahmen dieser Arbeit noch nicht entschieden werden.

7 N. Ri ehl, Ann. Physik 29, 636 [1937]; N. R i eh I u. M. Schön, Z. Physik 114, 682 [1939]; F. Möglich u. R. R om pe, Physik. Z. 41, 236 [1940].

\title{
Über die schwebstoff-abscheidende Wirkung von Polystyrol \\ Von Richard Weber*
}

(Z. Naturforschg. 1, 217-219 [1946]; eingegangen am 16. Februar 1946)

\begin{abstract}
Die Wirkung von Aerosolfiltern kann durch Imprägnierung der Filterfaser mit hochisolierenden organischen Kunststoffen wie Polystyrol gesteigert werden. Es ist dies ein elektrostatischer Effekt.
\end{abstract}

$\mathrm{M}$ ittels eines R e g e n er-Zerstäubers mit Spirale ${ }^{1}$ wurde ein Shellöl-Nebel erzeugt, der sich hinsichtlich Teilchengröß $\mathrm{e}^{2}$ und Massenkonzentration in reproduzierbarer Weise darstellen ließ und als Prüfnebel für Schwebstoffilter benutzt wurde. Die Prüfung geschah in derWeise, daß der Ölnebel durch Schwebstoffilter geleitet und die Konzentration der durch die Filter gegangenen Nebelteilchen tyndallometrisch gemessen wurde. Die geprüften Schweb-

* z. Zt. Dickschied über Bad Schwalbach (Taunus'). Die Versuche zu dieser Arbeit wurden im Laboratorium der A uer-Gesellschaft, Werk Oranienburg bei Berlin, durchgeführt. stoffilter wurden hergestellt durch Ablagernlassen von Zellstoffasern bzw. Lintersfasernstaub zu Faserschichten. Da dies auf maschinellem Wege geschah, konnten einheitliche Faserkuchen gefertigt werden. Der Druckunterschied an solchen in einem Filtertopf eingebauten Faserkuchen wurde

1 H. Freundlich, Kapillarchemie. II. Band, 787 [1932].

2 Bei dem Shellöl-Zerstäubernebel handelte es sich um einen nahezu isodispersen Nebel. Die Durchmesser der einzelnen Nebelteilchen lagen bei etwa $2,5 \times$ 10-5 $\mathrm{cm}$. Diese Ergebnisse können auf Grund umfassender eigener Untersuchungen an dem vorerwähnten Nebel als gesichert angesehen werden. 